\title{
LAPAROSCOPIC INCISIONAL/VENTRAL HERNIA REPAIR-TACKER VS. TRANSFACIAL MONOFILAMENT ABSORBABLE AND NON-ABSORBABLE SUTURE
}

\author{
Shailesh Kumar Katiyar1, ${ }^{\text {Pankaj Kapoor }}{ }^{2}$, Ashish Kumar Katiyar ${ }^{3}$ \\ 1 Professor, Department of Surgery, Rama Medical College, Mandhana, Kanpur. \\ ${ }^{2}$ Assistant Professor, Department of Surgery, Rama Medical College, Mandhana, Kanpur. \\ 3Junior Resident, Department of Surgery, Rama Medical College, Mandhana, Kanpur.
}

\section{ABSTRACT}

\section{BACKGROUND}

Incisional hernias are a common problem seen in approximately $11 \%$ of the patients after abdominal surgery. In case of Incisional/Ventral hernia repair, $50 \%$ or above of recurrence rates have been noticed. The advent of the laparoscopic repair of incisional and ventral hernias has brought down the recurrence rates to less than $10 \% .{ }^{[1]}$ The laparoscopic repair with the help of mesh fixation to the abdominal wall is poorly studied. This study was done to compare the various intraoperative and postoperative outcomes between two mesh fixation techniques, transfacial sutures alone versus tackers.

The aim of this study is to compare two techniques of mesh fixation, i.e. Tacker versus Transfacial monofilament absorbable and non-absorbable sutures alone for the laparoscopic repair of incisional/ventral hernia repair.

\section{MATERIALS AND METHODS}

This prospective randomised study was conducted from December 2013 to March 2015 in the Department of Surgery, Rama Medical College, Kanpur and Aastha Health Centre, Singhpur, Kanpur; 82 patients having incisional and primary ventral hernias consented for laparoscopic mesh under general anaesthesia. These patients were divided into two groups: Group I - Tacker mesh fixation (with four corner transfacial suture) and Group II - Suture alone mesh fixation. Various post-operative outcomes like pain, seroma formation, wound infection and recurrence were recorded.

\section{RESULTS}

The operative time was significantly higher in Group II. Patients in Group I felt more pain postoperatively as compared to Group II. The incidence of seromas was higher in Group II.

\section{CONCLUSION}

For small and medium sized defects in anatomically accessible areas, suture fixation is a better alternative than tacker fixation in terms of postoperative pain and cost.

\section{KEYWORDS}

Tacker, Mesh, Incisional Hernia, Transfacial Suture.

HOW TO CITE THIS ARTICLE: Katiyar SK, Kapoor P, Katiyar AK. Laparoscopic incisional/ventral hernia repair- Tacker vs. transfacial monofilament absorbable and non-absorbable suture. J. Evolution Med. Dent. Sci. 2017;6(2):97-99, DOI: 10.14260/Jemds/2017/24

\section{BACKGROUND}

Approximately, $11 \%$ of patients undergoing abdominal surgery develop incisional hernia. The traditional primary open repair of incisional hernias has shown to be associated with a recurrence rate of up to more than $60 \%$ and even with the prosthetic meshes a recurrence rate of up to $32 \%$ has been seen with long-term followup. In case of Incisional/Ventral hernia repair by open method, the chances of wound infection are more and further complications have been significantly increased.

The laparoscopic repair of incisional and ventral hernias brought a significant drop in the recurrence rate. It also adheres to the tension free repair principle by using a prosthetic mesh. During laparoscopic repair, the results are

Financial or Other, Competing Interest: None.

Submission 29-11-2016, Peer Review 24-12-2016,

Acceptance 30-12-2016, Published 05-01-2017.

Corresponding Author:

Dr. Shailesh Kumar Katiyar,

Singhpur, Kanpur.

E-mail: drskkatiyar59@rediffmail.com

DOI: $10.14260 /$ jemds $/ 2017 / 24$ better in terms of wound infection, postoperative hospital stay, recurrence rates and shorter operating times.[2]

The two most common method of mesh fixation are metallic tacks and transfacial sutures. Although, mesh fixation with tacks is convenient and time saving in the laparoscopic repair of incisional and ventral hernias, the tensile strength of a mesh fixed by transfacial sutures is greater than when fixed by tacks. ${ }^{[3,4]}$ Transfacial sutures penetrate all layers of the abdominal wall, thereby enable fixation of the mesh to the entire fasciomuscular layer of the abdominal wall. In our study, we compare the two most common methods of mesh fixation, i.e. tackers versus sutures.

\section{MATERIALS AND METHODS}

Patients admitted with incisional or primary ventral hernia for laparoscopic repair in Department of Surgery, Rama Medical College, Kanpur and Aastha Health Centre, Singhpur Kanpur, between December 2013 and March 2015 were divided into two groups. Group I - Tacker mesh fixation (with four corner transfacial sutures) or Group II - Suture mesh fixation. 


\section{Inclusion Criteria}

1. Smaller sized hernia defect (Less than $5 \times 5 \mathrm{~cm}$ ).

2. Primary ventral hernias/Umbilical hernias.

3. Incisional hernias not operated upon earlier.

\section{Exclusion Criteria}

1. Recurrent hernias.

2. Defect size greater than $5 \times 5 \mathrm{~cm}$.

3. Patients with skin infections and diabetes.

All patients received preoperative antibiotic prophylaxis. All patients were operated under general anaesthesia.

\section{Operative Technique}

In case of defect in the lower abdomen, the urinary bladder is decompressed by using Foley's catheter. After creation of pneumoperitoneum with a Veress needle at the umbilicus (For hernias in the lower abdomen) or left hypochondrium (Palmer's point) (For hernias through a midline incision through the umbilicus), a $12 \mathrm{~mm}$ laparoscopic port for a 30 degree telescope was introduced away from the left lateral margin of the defect in the left flank.

Additional two $5 \mathrm{~mm}$ ports were placed depending upon the location of the hernia defect. Omental and bowel adhesions were taken down using monopolar diathermy or scissors. The sac was left intact. The defect was identified and additional defects carefully looked for and defect size measured from outside. A mesh of appropriate size was used to overlap all the defects in a single piece with a margin of at least $4-5 \mathrm{~cm}$. Any deficient coverage at any margin was supplanted with an extra patch of mesh.

In Group I, mesh fixation was done with a $5 \mathrm{~mm}$ tacker (Protack, Autosuture, Tyco Healthcare, USA) along with 4 corner 1-0 polypropylene transfacial sutures. The tacks were placed at all four corners and then at $1-2 \mathrm{~cm}$ distance along the peripheral margin and again a second row of tacks were placed along the defect margin (Double crown technique).[5] In Group II, after the 4 corner 1-0 polypropylene transfacial sutures were tied subcutaneously, additional 1-0 polypropylene sutures were passed through the abdominal wall using a modified 16-G epidural needle and then the other end of the suture was grasped with a suture passer and brought outside through the abdominal wall. Sutures were tied outside subcutaneously.[6] The sutures were placed 2 - 3 $\mathrm{cm}$ apart. At the end of the procedure, pneumoperitoneum was decompressed and sheath at $12 \mathrm{~mm}$ ports closed with polyglactin 910 (Vicryl) 2-0. The operation time was measured from the skin incision given for Veress needle insertion till complete closure of all port sites.

\section{Post-Operative Care}

Patients were advised to use an abdominal binder in the postoperative period. After discharge from the hospital, the patients were followed in the Outpatient Clinic.

Ethical clearance for the study was obtained from the Institute Ethical Committee. The study subjects were included only after obtaining informed written consent. The study did not involve any form of experimental treatment.

\section{RESULTS}

82 patients were randomised into two groups with 41 patients in Group I and 41 in Group II. Incisional hernia was the most common type seen in both the groups followed by primary ventral hernias. Majority of the defects were seen in the infraumbilical region in both the groups. Heavyweight polypropylene was the most commonly used mesh followed by lightweight polypropylene polydioxanone-oxidised regenerated cellulose composite mesh. The operative time was significantly higher in Group II. Patients in Group I experience more pain post-operatively. The incidence of seromas was higher in Group II. The cost of surgery was significantly higher in Group I compared to Group II.

\section{DISCUSSION}

The most common complications of abdominal surgery by open method are incisional hernias. In case of incisional and primary ventral hernia repair, recurrence has been a major problem. With the introduction of new prosthetic materials for incisional hernia repair, there was a significant drop in the recurrence rates.[7] Burger et al have shown significantly lower cumulative 10-year recurrence rates with the use of prosthetic meshes.[8]

The Laparoscopic Approach for Incisional and Primary Ventral Hernia has Gained Popularity because of its

- Low recurrence rate.

- Short hospital stay.

- Reduced blood loss.

- Excellent cosmetic outcome.

- Low complication rate as compared to open repairs.

- To clearly define the margins of the hernia defect.

- To identify additional smaller "Swiss-cheese" defects, which can be missed in an open approach.

Although, laparoscopic incisional and ventral hernia repair has gained popularity, but there are many technical issues which have not been resolved yet. The issues of access to the abdominal cavity and mesh overlap size have more or less been resolved. Further, issues like the ideal mesh to be used, fixation technique, the necessity for closure of the defect before mesh fixation are areas of ongoing debate.

Currently, the most popular method of mesh fixation being used worldwide has been the use of tacks along with 4 corner transfacial sutures. The use of only transfacial suture fixation has not become very popular because of certain drawbacks. Suture fixation is time consuming and it is difficult to insert these transfacial sutures in certain anatomical areas like the pelvis, above the subcostal margin and laterally in the flanks.[9] Sometimes, there are chances of bowel injury during insertion of Suture Passer needle. There are also certain concern about the cosmetic appearance of these transfacial suture puncture sites over the skin of the abdominal wall after surgery. [10]

Variable recurrence rate have been reported in the literature with the use of different mesh fixation techniques.[11] In a collective review of 23 studies and 12 comparative studies by LeBlanc et al, mesh fixation with sutures only resulted in the lowest recurrence rate compared to that by tacks alone. Mesh fixation with tacks and sutures resulted in a recurrence rate of 3.5\%.[12] Though there has been a drop in recurrence rates with laparoscopic approach, factors which have been reported to be responsible for recurrences apart from technical errors like inadequate mesh overlap and inadequate fixation are, 
- Infection.

- Operator learning curve.

- Previous hernia repair.

- Obesity.

- $\quad$ Longer operating time.

- Larger defect.

- Intraoperative or postoperative complications.

However, there was no recurrence of hernia in the present study following mesh repair for incisional and ventral hernias.

\section{CONCLUSION}

Mesh fixation with transfacial sutures alone takes significantly more time than mesh fixation with tacks and four corner transfacial sutures. However, tacker fixation causes more pain in the postoperative period. There were no major intraoperative and postoperative complications. In case of suture fixation, the formation of seromas have been higher. All seromas settled with expectant management. The cost of the procedure was higher with the use of tacks. In conclusion for small and medium sized defects in anatomically accessible areas, suture fixation is a better alternative than tacker fixation in terms of postoperative pain and cost.

\section{REFERENCES}

[1] Agarwal BB, Agarwal S, Mahajan KC. Laparoscopic ventral hernia repair innovative anatomical closure, mesh insertion without $10 \mathrm{~mm}$ transmyofascial port, and atraumatic mesh fixation. Surg Endosc 2009;23(4):900-5.

[2] Barbaros U, Asoglu O, Seven R, et al. The comparison of laparoscopic and open ventral hernia repair. a prospective randomized study. Hernia 2007;11(1): 51-6.
[3] Berger D, Bientzle M, Muller A. prospective complication after laparoscopic incisional hernia repair. Incidence and treatment. Surg Endosc 2002;16:1720-3.

[4] Carbajo MA, Martp del Olmo JC, Blanco JI, et al. Laparoscopic approach to incisional hernia. Surg Endosc 2003;17(1):118-22.

[5] Carlson MA, Ludwig KA, Condon RE. Ventral hernia and other complications of 1000 midline incisions. South Med J 1995;88(4):450-3.

[6] Chowbey PK, Sharma A, Khullar R, et al. Laparoscopic ventral hernia repair. J Laparoendosc Adv Surg Tech A 2000;10(2):79-84.

[7] Franklin ME Jr, Gonzalez JJ, Glass JL, et al. Laparoscopic ventral and incisional hernia repair: an 11-year experience. Hernia 2004;8(1):23-7.

[8] Irvin TT, Stoddard CJ, Greaney MG, et al. Abdominal wound healing: a prospective clinical study. Br Med J 1977;2(6083):351-2.

[9] Kirshtein B, Lantsberg L, Avinoach E, et al. Laparoscopic repair of large incisional hernias. Surg Endosc 2002;16(12):1717-9.

[10] LeBlanc KA. Laparoscopic incisional hernia repair: are transfacial sutures necessary? A review of the literature. Surg Endosc 2007;21(4):508-13.

[11] Rutkow IM. Demographic and socioeconomic aspects of hernia repair in the United States in 2003. Surg Clin North Am 2003;83(5):1045-51.

[12] Usher FC. A new technique for repairing large abdominal wall defects. Arch Surg 1961;82:870-7. 